\title{
The Relationship between Dramatic Text and Performance with the Centrality of the Body with a Look at Anne Bogart's Rendition of The Persians
}

\author{
Samin Gheitasy ${ }^{1}$, Leila Montazeri² ${ }^{2}$, Simin Dolatkhah ${ }^{3}$ \\ ${ }^{1,2}$ Islamic Azad University, Science and Research Branch, Tehran \\ ${ }^{3}$ University of Tehran, Faculty of Visual Arts
}

\begin{abstract}
The dramatic text defines, to some extent, the structure of the work but the type of performance and the physical approach to the text can represent different meanings. The body of the actor, as a means of conveying concepts from the text to the audience, can be effective in creating different interpretations and meanings of the text. Since eons ago, directors have used the body of the actor with different approaches, and the application of body on the stage has always been underdoing changes. Anne Bogart is one of the few directors who is less known in the Iranian theater despite possessing the most updated and well-known methods of practice and performance in the world. Using her viewpoint method, she brings live and dynamic bodies to the stage; bodies that are able to convey the hidden meanings of the text to the audience in the most suitable way. The overall purpose of this research is to find the relationship between the dramatic text and the performance with the centrality of the body with a sociological view toward the body. To this end, by presenting Foucault's theories, the researchers defines the role of the body in the society and its extent of effectivity and impressibility. Finally, this study explores the implications of this role in each element of Aeschylus's The Persians, and it shall show how Bogart beautifully represents them using the bodies of her actors during performance.
\end{abstract}

Keywords: Anne Bogart, body, Michel Foucault, The Persians, play, sociology of body, theater 